\title{
Sinusite odontogênica: uma revisão de literatura
}

\author{
Odontogenic sinusitis: a literature review
}

\begin{abstract}
Carolina Oliveira de Lima, ${ }^{1}$ Karina Lopes Devito, ${ }^{1}$ Letícia Raquel Baraky Vasconcelos, ${ }^{2}$ Maíra do Prado, ${ }^{3}$ Celso Neiva Campos
'Departamento de Clínica Odontológica, Faculdade de Odontologia, Universidade Federal de Juiz de Fora, Juiz de Fora, MG, Brasil

2Departamento de Cirurgia, Faculdade de Medicina, Universidade Federal de Juiz de Fora, Juiz de Fora, MG, Brasil

${ }^{3}$ Faculdade de Odontologia, Centro de Saúde, Universidade Veiga de Almeida, Rio de Janeiro, RJ, Brasil

- Os autores declaram que não há conflito de interesse.
\end{abstract}

\section{Resumo}

Objetivo: realizar uma revisão de literatura sobre a anatomia e proximidade do seio maxilar com raízes dentárias, etiologia, microbiologia, sintomatologia, diagnóstico e tratamento das sinusites odontogênicas. Material e Métodos: foi realizada uma busca na literatura na base de dados PubMed, usando as palavras chave sinusite odontogênica, dente e seio maxilar. Trinta e seis artigos foram incluídos. Resultados: os artigos revisados sugerem que a iatrogenia e a periodontite apical são os principais fatores etiológicos da sinusite odontogênica. Os dentes mais próximos ao seio maxilar são os segundos pré-molares e molares superiores. Conclusão: a sinusite odontogênica é uma doença com etiologia, microbiologia e sintomatologia bem definidas. 0 diagnóstico errado pode levar a falhas no tratamento e sintomatologia persistente.

Palavras-chave: Sinusite odontogênica; Dente; Seio maxilar.

AbStRACT

Objective: to conduct a literature review about the anatomy and proximity of maxillary sinus with dental root canals, etiology, microbiology, symptoms, diagnosis, and treatment of odontogenic sinusitis. Material and Methods: a literature search was conducted in the PubMed database using the following keywords: odontogenic sinusitis, tooth and maxillary sinusitis. A total of 36 articles were found. Results: according to these data, the main cause of odontogenic sinusitis is iatrogenic cases and apical periodontitis. The second premolars and upper molars are closer to the maxillary. Conclusion: sinusitis odontogenic is a disease with well-defined etiology, microbiology, and symptoms. Misdiagnosis can lead to treatment failure and persistent symptoms.

Keywords: Odontogenic sinusitis; Tooth; Maxillary sinusitis.

\section{Introdução}

$\mathbf{0}$ seio maxilar é considerado o maior seio paranasal, sendo o primeiro a se desenvolver ${ }^{1}$ e está localizado na maxila, na região entre as cavidades orbital e nasal. Apresenta uma forma piramidal com a base voltada para a parede lateral da cavidade nasal e o ápice voltado para o processo zigomático da maxila. ${ }^{2}$

A inflamação da membrana sinusal que cobre a cavidade paranasal é referida como sinusite maxilar ${ }^{3}$ e sua origem é principalmente nasal. ${ }^{4}$ No entanto, em alguns casos, uma infecção dentária é um fator predisponente para o desenvolvimento da sinusite de origem dentária. A sinusite odontogênica corresponde aproximadamente por $10 \%$ a $12 \%$ dos casos de sinusite maxilar. ${ }^{1}$

A proximidade das raízes dos dentes maxilares posteriores com o seio maxilar associada à presença de inflamação, infecção ou iatrogenias de origem dentária pode afetar a integridade do assoalho do seio. ${ }^{5}$ Além disso, o desenvolvimento de uma lesão periapical em dentes cujos ápices da raiz estão perto ou estendendo-se para o seio maxilar pode provocar alterações inflamatórias no revestimento mucoso e, posteriormente, o desenvolvimento de sinusite odontogênica. ${ }^{6}$

Os sintomas da sinusite de origem odontogênica são semelhantes a não odontogênica, como obstrução ou congestão nasal, dor ou pressão na face e dores de cabeça. ${ }^{7.8}$ No entanto, quando a origem é odontogênica, geralmente, estes sintomas manifestam-se em apenas um lado da face. Outros sintomas como dor nos olhos, gotejamento pós-nasal, mau odor e dor de origem dentária também foram relatados. ${ }^{6}$
No entanto, apesar da sintomatologia semelhante, a sinusite odontogênica deve ser minunciosamente investigada pelo fato de diferir em relação à microbiologia, fisiopatologia e gerenciamento da sinusite de origem nasal. Assim, a definição da etiologia da doença é essencial para o tratamento adequado da sinusite odontogênica. ${ }^{9}$ Diante do exposto, o objetivo deste estudo é realizar uma revisão de literatura sobre anatomia e proximidade do seio maxilar com raízes dentárias, etiologia, microbiologia, sintomatologia, diagnóstico e tratamento das sinusites odontogênicas.

\section{Material e Métodos}

Foi realizada uma busca na literatura na base de dados PubMed, usando as palavras chave sinusite odontogênica, dente e seio maxilar. Trinta e seis artigos foram incluídos nesta pesquisa.

\section{Revisão de Literatura}

Esta revisão de literatura busca enfatizar diversos aspectos sobre o seio maxilar e a sinusite maxilar odontogênica como, a anatomia do seio maxilar; a proximidade dos dentes maxilares posteriores com o seio maxilar; associação de alterações dentárias com a sinusite maxilar e sintomas, diagnóstico, microbiologia e tratamento das sinusites de origem odontogênica.

\section{Anatomia do Seio Maxilar}

Os seios maxilares são cavidades ou compartimentos ósseos localizados dentro da maxila, acima dos dentes pré-molares e molares superiores, podendo se estender mais anteriormente até a região dos dentes caninos, porém não frequentemente. ${ }^{10} \mathrm{O}$ seu 
surgimento se dá por invaginações da cavidade nasal, a partir do terceiro mês de vida intrauterina, atingindo sua maturidade entre 12 e 14 anos de idade, momento que coincide com o período de erupção dos segundos molares superiores permanentes. ${ }^{6}$ Pode ser comparado com uma pirâmide triangular cuja base está orientada para a parede lateral da fossa nasal e cujo vértice corresponde à apófise zigomática do osso maxilar. ${ }^{5}$

Em exames de imagem radiográfica e tomografia computadorizada de feixe cônico (TCFC), o seio maxilar apresenta-se como uma área radiolúcida, de formato arredondado ou oval, devido à presença de ar no seu interior em condições normais, com uma camada radiopaca ao seu redor (figura 1 - seta vermelha). No entanto, na presença de sinusite maxilar, a imagem torna-se parcialmente ou totalmente radiopaca, devido à presença de espessamento mucoso do seio maxilar (figura 1 - seta azul). ${ }^{11}$

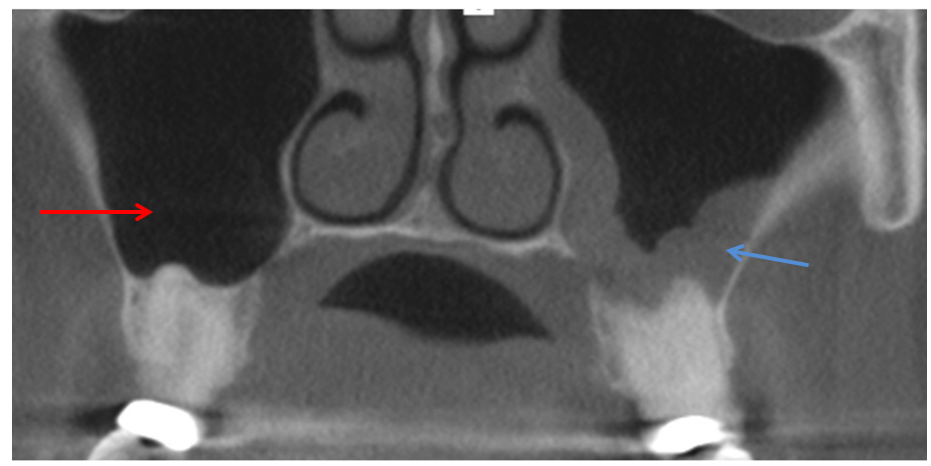

Figura 1. Exame tomográfico mostrando seio maxilar normal (seta em vermelho) e seio com sinusite (seta em azul)

\section{Proximidade dos Dentes com o Seio Maxilar}

Arx, Fodich e Bornstein ${ }^{12}$ avaliaram a proximidade das raízes dos pré-molares em relação ao seio maxilar, através da TCFC e observaram que as raízes palatinas dos primeiros pré-molares localizam-se mais perto do seio maxilar do que as raízes vestibulares. Já as raízes de segundos pré-molares posicionam-se mais próximas do seio maxilar do que as raízes de primeiros pré-molares. No entanto, a protrusão de raízes no interior do seio maxilar foi rara nos primeiros e segundos pré-molares.

Ok et al. ${ }^{13}$ avaliaram a relação entre as raízes dos pré-molares e molares superiores com o assoalho do seio maxilar através da TCFC. Os resultados mostraram que, em relação ao sexo, os homens apresentaram raízes que penetravam no seio maxilar (tipo 1), enquanto nas mulheres, a presença de raízes aquém do seio maxilar (tipo 3) foi o mais frequente. No que diz respeito à idade, pode-se observar que o tipo 1 (raízes no interior do seio maxilar) e tipo 2 (raízes em contato com a cortical, sem penetrar no interior do seio maxilar) decrescem com a idade ao contrário do tipo 3 . Observou-se o segundo pré-molar teve predominância do tipo 3. O primeiro molar teve predominância do tipo 1 e 2, já o segundo molar teve maior proximidade com o seio maxilar, quando comparado aos outros dentes. Fato que corrobora com os estudos de Pagin et al., ${ }^{14}$ e Kang, Kim e Kim ${ }^{15}$ que verificaram que os ápices das raízes mesiovestibulares de segundos molares superiores normalmente têm estreita proximidade com o assoalho do seio maxilar.

Estrela et $a l .{ }^{16}$ estudaram a relação anatômica entre os ápices dos dentes posteriores superiores e a mucosa do seio maxilar através da TCFC e observaram que a menor distância entre o assoalho do seio maxilar e os ápices dentários foi observada na raiz mesiovestibular do segundo molar e na raiz palatina do primeiro molar. A maior distância foi observada na raiz vestibular do primeiro pré-molar com diferença estatística significante. Observou-se que os primeiros pré-molares superiores não estão relacionados com o assoalho do seio maxilar.

\section{Alterações Dentárias e Sinusite Odontogênica}

Lechien et al. ${ }^{8}$ realizaram uma revisão sistemática sobre as causas da sinusite maxilar crônica odontogênica. Os resultados mostraram que $65,7 \%$ dos casos de sinusite odontogênica são de origem iatrogênica, já a presença de periodontite apical foi responsável por 16,8\% dos casos. Fato que está de acordo com o estudo de Lee e Lee ${ }^{17}$ onde complicações decorrentes de implantes dentários, por iatrogenia (37\%) e complicações decorrentes de exodontias foram as causas mais comuns $(29,6 \%)$, seguidos da periodontite apical (15\%).

Guerra-Pereira et al. ${ }^{18}$ averiguaram retrospectivamente 504 TCFC de pacientes de uma clínica privada. As TCFC foram realizadas para planejamentos cirúrgicos. As TCFC foram divididas em quatro grupos: IA-pacientes com fatores etiológicos de origem dental e espessamento mucoso (EM) superior a $2 \mathrm{~mm}$; IB - pacientes com fatores etiológicos de origem dental e com EM menor que $2 \mathrm{~mm}$; IIA - pacientes sem fatores etiológicos de origem dental e EM maior que $2 \mathrm{~mm}$ e IIB - pacientes sem fatores etiológicos de origem dental e com EM menor que $2 \mathrm{~mm}$. Observou-se que em $32,4 \%$ dos exames avaliados, os pacientes não tinham fatores etiológicos de origem dental e espessamento menor que $2 \mathrm{~mm}$. Os principais fatores etiológicos da sinusite maxilar odontogênica, de origem inflamatória ou infecciosa, foram tratamento endodôntico $(25,15 \%)$, lesões apicais $(20,43 \%)$, presença de cistos $(14,93 \%)$ e doença periodontal $(8,25 \%)$.

Sheik et al. ${ }^{19}$ verificaram a associação entre o espessamento mucoso e a perda óssea periodontal e a condição pulpoperiapical. Para tanto, foram avaliadas 180 TCFC, realizadas por distúrbios do desenvolvimento, doenças de seios paranasais, trauma e planejamento para implantes. A presença ou ausência do espessamento mucoso, perda óssea periodontal e a condição pulpoperiapical foram estabelecidas em escores para cada paciente. $\mathrm{O}$ espessamento mucoso foi observado em $39,4 \%$ dos pacientes e a perda óssea periodontal em 33\% dos pacientes. Observou-se que a presença de perda óssea e a condição pulpoperiapical estão associadas com a presença de espessamento mucoso, onde o efeito da perda óssea foi maior do que a condição pulpoperiapical.

Goller-Bulut et al. ${ }^{20}$ e de Lu et al. ${ }^{21}$ analisaram a relação entre o espessamento mucoso do seio maxilar, perda óssea periodontal e condição periapical dos dentes relacionados com o seio maxilar utilizando a TCFC. Os resultados mostraram que houve uma associação significativa entre a condição pulpoperiapical e o espessamento mucoso do seio maxilar. Junto a isso, a frequência do espessamento mucoso aumentou com a maior gravidade da lesão periapical. Fato que corrobora com Shanbhag et al. ${ }^{22}$ que avaliaram a frequência do espessamento da mucosa sinusal e a relação entre o espessamento com lesões periapicais e alterações periodontais em TCFC. Houve associação significativa entre o espes- 
samento da mucosa, sexo masculino, idade maior que 60 anos e dentes com lesões periapicais e doença periodontal. Observou-se que o espessamento da mucosa é mais visto em dentes com lesões periapicais e doença periodontal.

\section{Sintomas}

Os sintomas da sinusite de origem odontogênica são semelhantes a não odontogênica, como obstrução ou congestão nasal com presença de secreção amarelada, dor ou pressão na face, cefaleias que aumentam de intensidade com o movimento da cabeça, sensibilidade na região anterior da maxila e na região infraorbitária, dor nos olhos, gotejamento pós-nasal e mau odor. ${ }^{7,8}$ Os sintomas dentários variam desde uma dor aguda associada à inflamação pulpar até a dor proveniente de uma infecção dentária envolvendo o osso ao redor, como a doença periodontal e periodontite apical. No entanto, por ser de origem odontogênica, geralmente, estes sintomas manifestam-se em apenas um lado da face, ao contrário da sinusite não odontogênica. ${ }^{6}$

Devido à proximidade dos dentes maxilares posteriores com o seio maxilar, em casos de sinusite não odontogênica, a dor dentária e o aumento da sensibilidade pode estar presente em múltiplos dentes maxilares posteriores. Por isso, é importante o conhecimento da história prévia da sinusite maxilar e sua relação com tratamentos odontológicos pregressos. ${ }^{6}$

\section{Diagnóstico da Sinusite Odontogênica}

O diagnóstico da sinusite odontogênica envolve a realização de uma anamnese detalhada e cuidadosa e um exame físico extraoral e intraoral bem realizado. ${ }^{23} \mathrm{O}$ uso de exames por imagem pode fornecer informações valiosas no diagnóstico de sinusite maxilar odontogênica, como, por exemplo, uma imagem radiopaca no interior do seio maxilar, associada a corpos estranhos no seio, comunicações buco-sinusais ou inflamações e infecções oriundas de doenças periodontal e/ou endodôntica. ${ }^{24}$

No entanto, os exames utilizados rotineiramente na prática odontológica, como a radiografia periapical e panorâmica, não estimam a quantidade de perda óssea e não permitem a visualização completa do seio maxilar. ${ }^{25}$ Atualmente, a TCFC tem sido amplamente utilizada por cirurgiões-dentistas para avaliar os seios paranasais. ${ }^{26,27}$ A TCFC oferece uma baixa dose de radiação quando comparada a tomografia médica, menor tempo de aquisição e melhor resolução da imagem, ${ }^{28}$ permitindo ao clínico avaliar a relação entre alterações e/ou infecções dentárias com defeitos no assoalho do seio e qualquer alteração presente nos seios maxilares, em múltiplos planos. ${ }^{1,29}$

\section{Microbiologia}

De acordo com Brook ${ }^{6}$ os micro-organismos predominantemente isolados na sinusite maxilar crônica são Prevotella, Porphyromonas, Peptostreptococcus spp e Fusobacterium nucleatum. Estes achados confirmam que existe uma correlação entre a flora encontrada a nível periapical e encontrada no seio maxilar após a punção antral.

A alta taxa de micro-organismos anaeróbicos na sinusite odontogênica pode ser explicada devido à baixa drenagem e aumento da pressão intranasal que se desenvolve durante a inflamação. ${ }^{30}$ Isto pode reduzir a tensão de oxigênio no seio inflamado e com isso diminuir o fluxo sanguíneo da mucosa e a ação ciliar. A partir daí, as bactérias provenientes de infecções dentárias podem deslocar-se para dentro do seio maxilar devido à proximidade das raízes dos dentes posteriores maxilares com o assoalho do seio maxilar. ${ }^{13}$

\section{Tratamento da Sinusite Odontogênica}

O tratamento da sinusite odontogênica deve ser multidisciplinar e consiste primariamente na eliminação do fator causal de origem dentária, como por exemplo, extração dentária, tratamento endodôntico ou periodontal e remoção de corpos estranhos do interior do seio maxilar, acompanhada do manejo da infecção do seio maxilar através do uso de antibioticoterapia de largo espectro por 3 a 4 semanas e do uso de descongestionantes nasais. ${ }^{6}$

Além disso, otorrinolaringologistas têm utilizado a técnica endoscópica na associação do tratamento da sinusite maxilar odontogênica, que consiste na criação de uma loja óssea onde o tecido infectado, pólipos e corpos estranhos são removidos do interior do seio maxilar, após a remoção da causa dentária. ${ }^{4}$

Vale ressaltar que a não eliminação do fator causal irá provocar uma recidiva frequente onde o tratamento inadequado pode progredir para uma variedade de complicações, como: celulite orbitária, trombose do seio cavernoso, meningite, osteomielite, abscesso intracraniano e morte. ${ }^{31}$

Nair e Nair ${ }^{32}$ mostraram casos clínicos com alterações sinusais causadas por infecção odontogênica em tratamentos endodônticos mal sucedidos. Para isso, foram feitas TCFC dos três pacientes com alterações dentárias antes da intervenção odontológica e foi verificada a presença de uma área radiopaca no seio maxilar. Após a resolução das alterações dentárias houve uma regressão das alterações sinusais.

Cymerman et al. ${ }^{33}$ relataram o uso de TCFC em três casos clínicos para elucidar a patologia dental como fator etiológico da sinusite maxilar. Os pacientes apresentavam dor, congestão sinusal ou problemas respiratórios e a avaliação dos mesmos, foi feita por meio de radiografias periapicais e com TCFC. No primeiro caso, realizou-se a extração dos terceiros molares impactados e dos dentes 15 e 16. Nos outros pacientes, o tratamento endodôntico foi realizado. O acompanhamento radiográfico e com a TCFC foi feito com 6 e 12 meses após os procedimentos dentários. Todos os pacientes apresentaram a resolução da sinusite maxilar após o tratamento dentário adequado.

Abdala-Junior et al. ${ }^{34}$ mostraram um caso clínico de deslocamento de um implante dentário para o interior do seio maxilar. O paciente apresentava secreção nasal, dor e pressão na face. $\mathrm{Na}$ TCFC verificou-se a presença do implante dentário dentro do seio maxilar associado a dois cistos de retenção mucosos. $\mathrm{O}$ tratamento foi a remoção cirúrgica do implante através da técnica de Caldwell Luc, que consiste no acesso ao seio maxilar, através de uma incisão na altura da fossa canina com abertura acima dos ápices dos dentes, antibioticoterapia por oito dias e uso de descongestionante nasal. Após 15 meses de controle, o paciente encontra-se sem ocorrências sinusais ou queixa sintomatológica.

Manzi et al. ${ }^{35}$ relatam um caso clínico onde a paciente queixava-se de pressão interna, sensação de cabeça pesada do lado direito e dificuldade de respiração pela narina direita, com história de sinusite recorrente. Na TCFC observou-se a presença de imagem 
radiopaca no seio maxilar direito e lesão de furca extensa, com perda da cortical do assoalho do seio maxilar. Após a exodontia do dente 16 e prescrição de antibióticos, observou-se remissão da sintomatologia e da região radiopaca associada ao seio maxilar.

\section{Resultados}

As principais causas da sinusite odontogênica são as iatrogenias, a periodontite apical e a doença periodontal, como descrito na Tabela 1.

Os dentes superiores posteriores (pré-molares e molares) mais próximos a cortical do seio maxilar estão descritos na Tabela 2.

Tabela 1. Causas da sinusite odontogênica

\begin{tabular}{l|l}
\hline \multicolumn{1}{c|}{ Autor } & \multicolumn{1}{c}{ Etiologia } \\
\hline Lechien et al. $^{8}$ & iatrogenia \\
\hline Lee e Lee $^{17}$ & $\begin{array}{l}\text { iatrogenia decorrente de implantes } \\
\text { dentários }\end{array}$ \\
\hline Guerra-Pereira et al. ${ }^{18}$ & tratamento endodôntico \\
\hline Sheik et al. ${ }^{19}$ & doença periodontal e lesão periapical \\
\hline Goller-Bulut et al. ${ }^{20}$ & lesão periapical \\
\hline Lu et al. ${ }^{21}$ & lesão periapical \\
\hline Shanbhag et al. ${ }^{22}$ & doença periodontal e lesão periapical \\
\hline
\end{tabular}

Tabela 2. Dentes/raízes dos dentes superiores mais próximas do seio maxilar

\begin{tabular}{l|l}
\hline \multicolumn{1}{c|}{ Autor } & $\begin{array}{l}\text { Dente/raiz mais próximo do seio } \\
\text { maxilar }\end{array}$ \\
\hline Arx, Fodich e Bornstein ${ }^{12}$ & segundo pré-molar \\
\hline OK et al. ${ }^{13}$ & segundo molar \\
\hline Pagin et al. ${ }^{14}$ & $\begin{array}{l}\text { raiz mesiovestibular do segundo } \\
\text { molar }\end{array}$ \\
\hline Estrela et al. ${ }^{16}$ & $\begin{array}{l}\text { raiz mesiovestibular do segundo } \\
\text { molar e palatina do primeiro } \\
\text { molar }\end{array}$ \\
\hline Kang, Kim e Kim ${ }^{15}$ & $\begin{array}{l}\text { raiz mesiovestibular do segundo } \\
\text { molar }\end{array}$ \\
\hline
\end{tabular}

\section{Discussão}

A sinusite maxilar odontogênica corresponde de $10-12 \%$ dos casos de sinusite maxilar., ${ }^{1,2}$ No entanto, outros estudos relatam que essa prevalência chega a até $40 \%,{ }^{28,36}$ o que mostra a importância do conhecimento da etiologia, diagnóstico e tratamento desta patologia.

Diversos estudos mostram que o segundo molar é o dente com maior proximidade com o seio maxilar, seguido do primeiro molar e segundo pré-molar. ${ }^{12-16}$ Entretanto, não existem estudos que associem a proximidade dos dentes superiores posteriores com o seio maxilar em pacientes diagnosticados com sinusite maxilar.

Estudos mostram que a etiologia da sinusite maxilar odontogênica está relacionada com complicações/iatrogenias decorrentes de implantes dentários, e de exodontias, como a colocação do implante no seio maxilar (figura 2), rompimento da cortical e comunicação buco-sinusal durante exodontia, seguida da periodontite apical (figura 3)..$^{18-22}$ No entanto, estes estudos, avaliaram TCFC de pacientes em bancos de dados, que realizaram os exames por diferentes fins, como distúrbios do desenvolvimento, suspeitas de fratura radicular, trauma e planejamento para implantes ${ }^{18-20,25-27}$ e não exames de pacientes diagnosticados com sinusite maxilar.

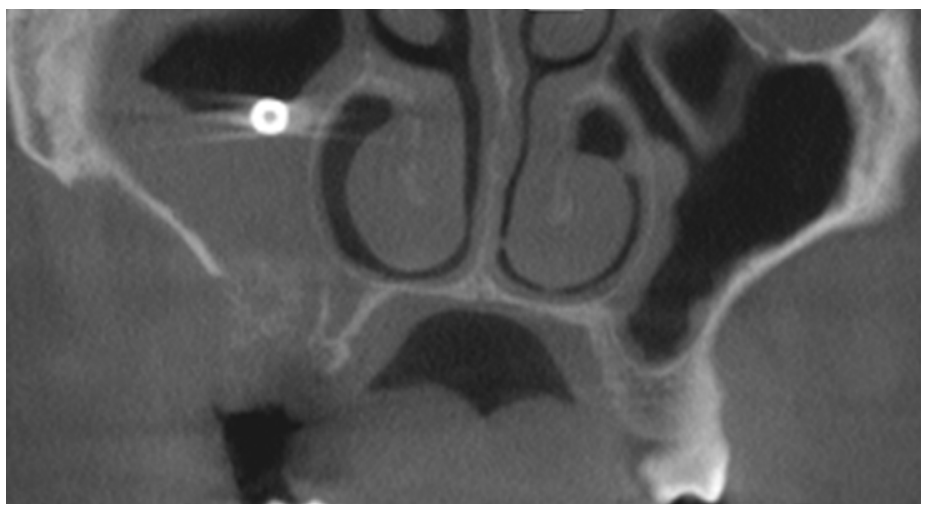

Figura 2. Exame tomográfico de paciente com sinusite maxilar e implante dentro do seio maxilar

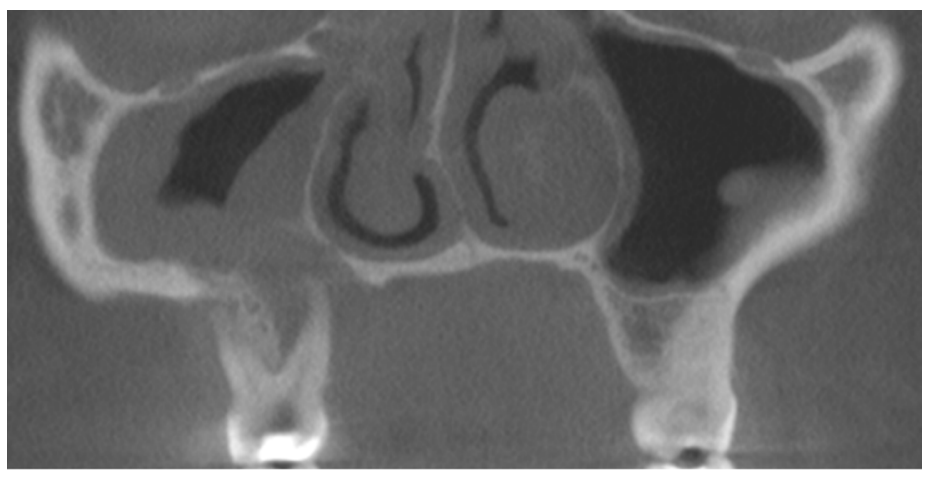

Figura 3. Exame tomográfico de paciente com sinusite maxilar e imagem radiolúcida associada ao ápice do dente 16

Além disso, a definição de sinusite maxilar difere entre os estudos de acordo com o grau de espessamento mucoso. ${ }^{20-23} \mathrm{Al}-$ guns estudos citam que a sinusite maxilar só deve ser considerada quando o espessamento mucoso é maior do que $2 \mathrm{~mm},{ }^{18,22}$ enquanto outros mostram que qualquer espessamento caracteriza a sinusite maxilar. ${ }^{19,20,29}$ Esta variação, apesar de pequena, pode gerar viés no diagnóstico da sinusite, sobre ou subestimando a prevalência real da sinusite maxilar.

Vale ressaltar que os estudos presentes na literatura avaliaram apenas os exames de TCFC, ${ }^{18-22,24-26}$ sem levar em conta os possíveis sinais e sintomas que seriam encontrados durante uma avaliação clínica do paciente.

Por isso, são necessários novos estudos que levem em conta os achados tomográficos e a associação destes achados com a avaliação clínica do paciente.

\section{Conclusão}

A etiologia da sinusite odontogênica são as iatrogenias em procedimentos odontológicos seguida da periodontite apical. Os segundos pré-molares e os molares superiores têm maior proximidade com a cortical do seio maxilar. Há uma correlação entre a flora encontrada em nível periapical e encontrada no seio maxilar após a punção antral. $\mathrm{O}$ diagnóstico da sinusite odontogênica envolve o exame clínico e o uso de imagens de TCFC e o seu tratamento consiste, primariamente, na eliminação do fator causal de origem dentária. 


\section{Referências}

1. Mehra P, Murad H. Maxillary sinus disease of odontogenic origin. Otolaryngol Clin North Am. 2004;37:347-64.

2. Mehra P, Jeong D. Maxillary sinusitis of odontogenic origin. Curr Infect Dis Rep. 2008;10(3):205-10.

3. Melén I, Lindahl L, Andréasson L, Rundcrantz H. Chronic maxillary sinusitis. Definition, diagnosis and relation to dental infections and nasal polyposis. Acta Otolaryngol. 1986;101(3-4):320-7.

4. Lopatin AS, Sysolyatin SP, Sysolyatin PG, Melnikov MN. Chronic maxillary sinusitis of dental origin: is external surgical approach mandatory? Laryngoscope. 2002;112(6):1056-9.

5. Maloney PL, Doku HC. Maxillary sinusitis of odontogenic origin. J Can Dent Assoc. 1968;34(11):591-603.

6. Brook I. Sinusits of odontogenic origin. Otolaryngol Head Neck Surg. 2006; 135(3):349-55.

7. Arias-Irimia O, Barona-Dorado C, Santos-Marino JA, Martínez-Rodríguez M, Martínez-González JA. Meta-analisis of the etiology of odontogenic maxillary sinusitis. Med Oral Patol Oral Cir Bucal. 2010;15(1):70-3.

8. Lechien JR, Filleul O, Araujo PC, Hsieh JW, Chantrain G, Saussez S. Chronic Maxillary Rhinosinusitis of Dental Origin: A Systematic Review of 674 Patient Cases. Int J Otolaryngol. 2014; 2014:1-9.

9. Simuntis R, Kubilius R, Vaitkus S. Odontogenic maxillary sinusitis: A review Stomatologija, Baltic Dental Maxilofac J. 2014;16(2):39-43.

10. Ferreira JRM, Vidigal Jr GMV, Cardoso ES. Considerações anatômicas relacionadas à cirurgia do seio maxilar. Implantnews. 2007;4(2):159-63.

11. Arieta LC, Silva MAA, Rockenbach MIB, Veeek EB. Extensões dos seios maxilares detectada em radiografias periapicais. Ver Odonto Cienc. 2005;20(47):18-22. 12. Arx TV, Fodich I, Bornstein MM. Proximity of Premolar Roots to Maxillary Sinus: A Radiographic Survey Using Cone-beam Computed Tomography. J Endod. 2014;40(10):1541-8.

13. Ok E, Gungor E, Colak M, Altunsoy M, Nur BG, Aglarci OS. Evaluation of the relationship between the maxillary posterior teeth and the sinus floor using cone-beam computed tomography. Surg Radiol Anat. 2014;26(9):907-14

14. Pagin O, Centurion BS, Rubira-Bullen IRF, Capelozza ALA. Maxillary Sinus and Posterior Teeth: Accessing Close Relationship by Cone-beam Computed Tomographic Scanning in a Brazilian Population. J Endod. 2013;39(6):748-51.

15. Kang SH, Kim BS, Kim Y. Proximity of Posterior Teeth to the Maxillary Sinus and Buccal Bone Thickness: A Biometric Assessment Using Cone-beam Computed Tomography J Endod. 2015;41(11):1839-46.

16. Estrela C, Nunes CABCM, Guedes O, Alencar AHG, Estrela CRA, Silva RA, et al. Study of Anatomical Relationship between Posterior Teeth and Maxillary Sinus Floor in a Subpopulation of the Brazilian Central Region Using Cone- Beam Computed Tomography - Part 2. Braz Dental J. 2016;27(1):9-15.

17. Lee KC, Lee SJ. Clinical features and treatments of odontogenic sinusitis. Yonsei Med J. 2010;51(6):932-7.

18. Guerra-Pereira I, Vaz P, Faria-Almeida R, Braga AC, Felino A. CT maxillary sinus evaluation-A retrospective cohort study. Med Oral Patol Oral Cir Bucal. 2015;1(20):e419-26.

19. Sheikhi M, Pozve NJ, Khorrami L. Using cone beam computed tomography to detect the relationship between the periodontal bone loss and mucosal thickening of the maxillary sinus. Dent Res J (Isfahan). 2014;11(4):495-501.

20. Goller-Bulut D, Sekerci AE, Kose E, Sisman Y. Cone beam computed tomo- graphic analysis of maxillary premolars and molars to detect the relationship between periapical and marginal bone loss and mucosal thickness of maxillary sinus. Med Oral Patol Oral Cir Bucal. 2015;20(5):e572-9.

21. Lu Y, Liu Z, Zhang L, Zhou X, Zheng Q, Duan X, et al. Associations between Maxillary Sinus Mucosal Thickening and Apical Periodontitis Using Cone-Beam Computed Tomography Scanning: A Retrospective Study. Endod. 2012;38(8):1069-74.

22. Shanbhag S, Karnik P, Shirke P, Shanbhag V. Association between Periapical Lesions and Maxillary Sinus Mucosal Thickening: A Retrospective Cone-beam Computed Tomographic Study. J Endod. 2013;39(7):853-7.

23. Low KMT, Dula K, Burgin W, Arx TV. Comparison of periapical radiography and limited cone-beam tomography in posterior maxillary teeth referred for apical surgery. J Endod. 2008;34(5):557-62.

24. Ueda M, Kaneda T. Maxillary sinusitis caused by dental implants. J. Oral Maxillofac. Surg. 1992;50(3):285-7.

25. Oberli K, Bornstein MM, Von Arx T. Periapical surgery and the maxillary sinus: radiographic parameters for clinical outcome. Oral Surg Oral Med Oral Pathol Oral Radiol Endod. 2007;103(6):848-53.

26. Bremke M, Sesterhenn AM, Murthum T, Al Hail A, Bien S, Werner JA. Digital volume tomography (DVT) as a diagnostic modality of the anterior skull base. Acta Otolaryngol. 2009;129(10):1106-14.

27. Cakli H, Cingi C, Ay Y, Oghan F, Ozer T, Kaya E. Use of cone beam computed tomography in otolaryngologic treatments. Eur Arch Otorhinolaryngol. 2012;269(3):711-20.

28. Suomalainen A, Kiljunen T, Kaser Y, Peltola J, Kortesniemi M. Dosimetry and image quality of four dental cone beam computed tomography scanners compared with multislice computed tomography scanners. Dentomaxillofac Radiol. 2009;38(60):367-78.

29. Maillet M, Bowles WR, McClanahan SL, John MT, Ahmad M. Conebeam Computed Tomography Evaluation of Maxillary Sinusitis. J Endod. 2011;37(6):753-7.

30. Carenfelt C, Lundberg C. Purulent and non-purulent maxillary sinus secretions with respect to pO2, pCO2 and pH. Acta Otolaryngol. 1977;84(1-2):138-44. 31. Schow SR. Doenças Odondogénicas do Seio Maxilar. In: Peterson LJ, Ellis E, Hupp JR, Tucker MR. Cirurgia Oral e Maxilofacial Contemporânea. 4th ed. Rio de Janeiro: Elsevier. 2005:443-59.

32. Nair UM, Nair MK. Maxillary sinusitis of odontogenic origin: cone-beam volumetric computerized tomography-aided diagnosis. Oral Surg Oral Med Oral Pathol Oral Radiol Endod. 2010;110(6):e53-7.

33. Cymerman JJ, Cymerman DH, O'Dwyer RS. Evaluation of Odontogenic Maxillary Sinusitis Using Cone-Beam Computed Tomography: Three Case Reports. J Endod. 2011;37(10):1465-9.

34. Abdala Junior R, Cavezzi Junior O. Displacement of dental implant to the maxillary sinus: Case report. Rev Port Estomatol Med Dent Cir Maxilofac. 2013;54(4):228-33.

35. Manzi FR, Dias DRCM, Bustamante RPC, Villoria EM, Peyneau PD, Cardoso CAA. Tomographic diagnosis and treatment of odontogenic sinusitis: a case report. Arq Bras de Odont. 2013;9(2):28-34.

36. Patel NA, Ferguson BJ. Odontogenic sinusitis: an ancient but under-appreciated cause of maxillary sinusitis. Curr Opin Otolaryngol Head Neck Surg. 2012;20(1):24-8.

\section{Mini Currículo e Contribuição dos Autores}

1. Carolina Oliveira de Lima - cirurgiã-dentista e mestranda. Contribuição: levantamento dos artigos, interpretação dos dados, preparação e redação do manuscrito.

2. Karina Lopes Devito - cirurgiã-dentista e PhD. Contribuição: colaboração científica e intelectual efetiva para o estudo e revisão crítica.

3. Letícia Raquel Baraky Vasconcelos - cirurgiã-dentista e PhD. Contribuição: colaboração científica e intelectual efetiva para o estudo e revisão crítica.

4. Maíra do Prado - cirurgiã-dentista e PhD. Contribuição: colaboração científica e intelectual efetiva para o estudo; concepção e delineamento; revisão crítica e aprovação final.

5. Celso Neiva Campos - cirurgião-dentista e PhD. Contribuição: colaboração científica e intelectual efetiva para o estudo; concepção e delineamento; revisão crítica e aprovação final.

Recebido em: 14/12/2016 / Aprovado em: 04/02/2017

Autor Correspondente

Maíra do Prado

E-mail: mairapr@hotmail.com 